\title{
Prognosis of hepatic epithelioid hemangioendothelioma after living donor liver transplantation
}

\author{
Byeong-Gon NA, Shin HWANG*, Chul-Soo AHN, Deok-Bog MOON, Tae-Yong HA, \\ Gi-Won SONG, Dong-Hwan JUNG, Gil-Chun PARK, Young-In YOON, Sung-Gyu LEE
}

Department of Surgery, Asan Medical Center, University of Ulsan College of Medicine, Seoul, Korea

Introduction: Epithelioid hemangioendothelioma (EHE) is a rare borderline vascular tumor. Due to its rarity and protean behavior, the optimal treatment of hepatic EHE has not yet been standardized. This single-center study describes outcomes in patients with hepatic EHE who underwent living donor liver transplantation (LDLT).

Methods: The medical records of patients who underwent LDLT for hepatic EHE from 2007 to 2016 were reviewed.

Results: During 10-year period, four patients, one man and three women, of mean age $41.3 \pm 11.1$ years, underwent LDLT for hepatic EHE. Based on imaging modalities, these patients were preoperatively diagnosed with EHE or hepatocellular carcinoma, with percutaneous liver biopsy confirming that all four had hepatic EHE. The tumors were multiple and scattered over entire liver, precluding liver resection. Blood tumor markers were not elevated, except that CA19-9 and des- $\gamma$-carboxy prothrombin was slightly elevated in one patient. Mean model for end-stage liver disease score was $10.8 \pm 5.7$. All patients underwent LDLT using modified right liver grafts, with graft-recipient weight ratio of $1.11 \pm 0.19$, and all recovered uneventfully after LDLT. One patient died due to tumor recurrence at 9 months, whereas the other three have done well without tumor recurrence, resulting in 5-year disease-free and overall patient survival rates of $75 \%$ each. The patient with tumor recurrence was classified as a high-risk patient based on the original and modified hepatic EHE-LT scoring systems.

Conclusions: LDLT can be an effective treatment for patients with unresectable hepatic EHEs that are confined within the liver and absence of macrovascular invasion and lymph node metastasis. 